\title{
Oscillation of Solutions and Their Arbitrary Order Derivatives of Higher Order Non-Homogeneous LDE
}

\author{
M. Saidani, B. Belaïdi
}

\begin{abstract}
In this paper, we investigate the iterated order of solutions of higher order nonhomogeneous linear differential equations

$$
A_{k}(z) f^{(k)}+A_{k-1}(z) f^{(k-1)}+\cdots+A_{1}(z) f^{\prime}+A_{0}(z) f=F(z),
$$

where $A_{0}(z), A_{1}(z), \cdots, A_{k}(z) \not \equiv 0$ are entire functions of finite iterated $p$-order and $F(z)$ is entire function of infinite iterated $p$-order. We improve and extend some recent results of Chen and we obtain general estimates of the iterated convergence exponent, the iterated $p$-order and the iterated convergence exponent of arbitrary order derivatives of the solutions of the above equation.
\end{abstract}

Keywords: Linear differential equations, Entire function, Iterated order.

\section{Introduction and main results}

In this paper, we shall assume that the reader is familiar with the fundamental results and the standard notations of the Nevanlinna value distribution theory of meromorphic functions see ([10], [13], [22]), such as $T(r, f), N(r, f), m(r, f)$. For the definition of iterated order of meromorphic function, we use the same definition as in [15], [16]. For all $r \in \mathbb{R}$, we define $\exp _{1} r:=e^{r}$ and $\exp _{p+1} r:=\exp \left(\exp _{p} r\right), p \in \mathbb{N}$. We also define for all $r \in(0,+\infty)$ sufficiently large $\log _{1} r:=\log r$ and $\log _{p+1} r:=\log \left(\log _{p} r\right), p \in \mathbb{N}$.

Definition 1.1 $([15],[16])$ Let $f$ be a meromorphic function. Then the iterated $p$-order $\rho_{p}(f)$ of $f$ is defined by

$$
\rho_{p}(f):=\limsup _{r \rightarrow+\infty} \frac{\log _{p} T(r, f)}{\log r},(p \geq 1 \text { is an integer }) .
$$

Manuscript received August 11, 2017; accepted November 20, 2017.

M. Saidani, B. Belaïdi (corresponding author) are with Department of Mathematics, Laboratory of Pure and Applied Mathematics, University of Mostaganem, Algeria 
For $p=1$, this notation is called order and for $p=2$ hyper-order. If $f$ is an entire function, then the iterated $p$-order $\rho_{p}(f)$ of $f$ is defined by

$$
\rho_{p}(f):=\limsup _{r \rightarrow+\infty} \frac{\log _{p} T(r, f)}{\log r}=\limsup _{r \rightarrow+\infty} \frac{\log _{p+1} M(r, f)}{\log r},(p \geq 1 \text { is an integer })
$$

where $M(r, f)=\max _{|z|=r}|f(z)|$.

Definition 1.2 [15] The finiteness degree of the order of a meromorphic function $f$ is defined by

$$
i(f):=\left\{\begin{array}{c}
0, \quad \text { for } f \text { rational, } \\
\min \left\{j \in \mathbb{N}: \rho_{j}(f)<+\infty\right\}, \text { for } f \text { transcendental for which } \\
\text { some } j \in \mathbb{N} \text { with } \rho_{j}(f)<+\infty \text { exists, } \\
+\infty, \quad \text { for } f \text { with } \rho_{j}(f)=+\infty, \forall j \in \mathbb{N} .
\end{array}\right.
$$

Definition 1.3 [15] Let $n(r, a)$ be the unintegrated counting function for the sequence of $a$-points of a meromorphic function $f$. Then the iterated convergence exponent of $a$-points of $f$ is defined by

$$
\lambda_{p}(f, a):=\limsup _{r \rightarrow+\infty} \frac{\log _{p} n(r, a)}{\log r},(p \geq 1 \text { is an integer }) .
$$

In the definition of the iterated convergence exponent, we may replace $n(r, a)$ with the integrated counting function $N(r, a)$, and we have

$$
\lambda_{p}(f, a):=\limsup _{r \rightarrow+\infty} \frac{\log _{p} N(r, a)}{\log r},(p \geq 1 \text { is an integer }),
$$

where $N(r, a)=N(r, a, f)=N\left(r, \frac{1}{f-a}\right)$. If $a=0$, then the iterated convergence exponent of the zero-sequence is defined by

$$
\lambda_{p}(f):=\limsup _{r \rightarrow+\infty} \frac{\log _{p}^{+} N\left(r, \frac{1}{f}\right)}{\log r},(p \geq 1 \text { is an integer }),
$$

where $N\left(r, \frac{1}{f}\right)$ is the integrated counting of zeros of $f$ in $\{z:|z| \leq r\}$. Similarly, if $a=\infty$, then the iterated convergence exponent of the pole-sequence is defined by

$$
\lambda_{p}\left(\frac{1}{f}\right):=\limsup _{r \rightarrow+\infty} \frac{\log _{p} N(r, f)}{\log r},(p \geq 1 \text { is an integer }) .
$$


Definition 1.4 [15] Let $f$ be a meromorphic function. Then, the iterated exponent of convergence of the sequence of distinct zeros of $f$ is defined as

$$
\bar{\lambda}_{p}(f):=\limsup _{r \rightarrow+\infty} \frac{\log _{p} \bar{N}\left(r, \frac{1}{f}\right)}{\log r} \quad(p \geqslant 1 \text { is an integer }),
$$

where $\bar{N}\left(r, \frac{1}{f}\right)$ is the integrated counting function of distinct zeros of $f$ in $\{z:|z| \leq r\}$. For $p=1$, this notation is called exponent of convergence of the sequence of distinct zeros and for $p=2$ hyper-exponent of convergence of the sequence of distinct zeros.

Definition 1.5 [15] The finiteness degree of the iterated convergence exponent is defined by

$$
i_{\lambda}(f, a):=\left\{\begin{array}{c}
0, \quad \text { if } n(r, a)=O(\log r), \\
\min \left\{j \in \mathbb{N}: \lambda_{j}(f, a)<+\infty\right\}, \text { for some } j \in \mathbb{N} \\
\text { with } \lambda_{j}(f, a)<+\infty \text { exists, } \\
+\infty, \quad \text { if } \lambda_{j}(f, a)=+\infty \text { for all } j \in \mathbb{N} .
\end{array}\right.
$$

Remark 1.1. If $a=0$, then we set $i_{\lambda}(f, a)=i_{\lambda}(f)$. If $a=\infty$, then we set $i_{\lambda}(f, a)=i_{\lambda}\left(\frac{1}{f}\right)$. Similarly, we can define $i_{\bar{\lambda}}(f, a)=i_{\bar{\lambda}}(f)$ if $a=0$ and $i_{\bar{\lambda}}(f, a)=i_{\bar{\lambda}}\left(\frac{1}{f}\right)$ if $a=\infty$.

Definition 1.6 [14], [20] The iterated lower $p$-order $\mu_{p}(f)$ of a meromorphic function $f$ is defined by

$$
\mu_{p}(f):=\liminf _{r \rightarrow+\infty} \frac{\log _{p} T(r, f)}{\log r},(p \geq 1 \text { is an integer }) .
$$

The Lebesgue linear measure of a set $E \subset[0,+\infty)$ is $m(E)=\int_{E} d t$, and the logarithmic measure of a set $F \subset(1,+\infty)$ is $m_{l}(F)=\int_{F} \frac{d t}{t}$. The upper density of $E \subset[0,+\infty)$ is given by

$$
\overline{\operatorname{dens}}(E)=\limsup _{r \rightarrow+\infty} \frac{m(E \cap[0, r])}{r}
$$

and the upper logarithmic density of the set $F \subset(1,+\infty)$ is defined by

$$
\overline{\log \operatorname{dens}}(F)=\limsup _{r \longrightarrow+\infty} \frac{m_{l}(F \cap[1, r])}{\log r} .
$$

Proposition 1.1 [3] For all $H \subset(1,+\infty)$ the following statements hold:

(i) If $m_{l}(H)=+\infty$, then $m(H)=+\infty$;

(ii) If $\overline{\text { dens }}(H)>0$, then $m(H)=+\infty$;

(iii) If $\overline{\log \operatorname{dens}}(H)>0$, then $m_{l}(H)=+\infty$. 
For $k \geq 2$, we consider the homogeneous and non-homogeneous linear differential equations

$$
f^{(k)}+\sum_{j=1}^{k-1} A_{j} f^{(j)}+A_{0} f=0
$$

and

$$
f^{(k)}+\sum_{j=1}^{k-1} A_{j} f^{(j)}+A_{0} f=F
$$

where $A_{0}(z) \not \equiv 0, A_{1}(z), \cdots, A_{k-1}(z), F(z) \not \equiv 0$ are entire functions. It is well-known that if some of the coefficients of the linear differential equation (1.1) are transcendental then the equation (1.1) has at least one solution of infinite order. Thus, the question which arises is : What conditions on $A_{0}(z) \not \equiv 0, A_{1}(z), \cdots, A_{k-1}(z)$ will guarantee that every solution $f \not \equiv 0$ of (1.1) has an infinite order? For the above question, there are many results for the second and higher order linear differential equations (see for example [1], [2], [7], [12]). When $A_{0}(z) \not \equiv 0, A_{1}(z), \cdots, A_{k-1}(z)$ and $F(z) \not \equiv 0$ are meromorphic functions, a precise estimation of the hyper-order of meromorphic solutions of the above equations has been given by Long and Zhu in [17], where they provided that there exists one dominant coefficient. Recently, in [18] the authors improved the previous results by studying the growth of meromorphic solutions of

$$
\begin{gathered}
A_{k}(z) f^{(k)}+A_{k-1}(z) f^{(k-1)}+\cdots+A_{1}(z) f^{\prime}+A_{0}(z) f=0, \\
A_{k}(z) f^{(k)}+A_{k-1}(z) f^{(k-1)}+\cdots+A_{1}(z) f^{\prime}+A_{0}(z) f=F(z)
\end{gathered}
$$

and obtained the following results.

Theorem $\mathbf{A}$ [18] Let $H$ be a set of complex numbers satisfying $\overline{\log \text { dens }}\{|z|: z \in H\}>0$ (or $\left.m_{l}(\{|z|: z \in H\})=+\infty\right)$ and let $A_{j}(z)(j=0,1, \cdots, k)$, be entire functions such that $A_{k}(z) \not \equiv 0$. Suppose there exists an integer $s, 0 \leq s \leq k$ such that $i\left(A_{s}\right)=p, 0<p<+\infty$, and

$$
\max \left\{\rho_{p}\left(A_{j}\right), j \neq s, j=0,1, \cdots, k\right\}<\mu_{p}\left(A_{s}\right) \leq \rho_{p}\left(A_{s}\right)<+\infty
$$

( $p \geq 1$ is an integer) and for some constants $0 \leq \beta<\alpha$, we have, for all $\varepsilon>0$ sufficiently small,

$$
\begin{gathered}
\left|A_{j}(z)\right| \leq \exp _{p}\left(\beta|z|^{\rho_{p}\left(A_{s}\right)-\varepsilon}\right), j \neq s, j=0,1, \cdots, k, \\
\left|A_{s}(z)\right| \geq \exp _{p}\left(\alpha|z|^{\rho_{p}\left(A_{s}\right)-\varepsilon}\right),
\end{gathered}
$$

as $z \rightarrow \infty$ for $z \in H$. Then every transcendental meromorphic solution $f$ of equation (1.3) with $\lambda_{p}\left(\frac{1}{f}\right)<\mu_{p}(f)$ satisfies $i(f)=p+1$ and $\rho_{p+1}(f)=\rho_{p}\left(A_{s}\right)$. 
Theorem $\mathbf{B}[18]$ Let $H$ be a set of complex numbers satisfying $\overline{\log \text { dens }}\{|z|: z \in H\}>0$ $\left(\right.$ or $\left.m_{l}(\{|z|: z \in H\})=+\infty\right)$ and let $A_{j}(z)(j=0,1, \cdots, k), F(z) \not \equiv 0$ be entire functions such that $A_{k}(z) \not \equiv 0$. Suppose there exists an integer $s, 0 \leq s \leq k$ such that $i\left(A_{s}\right)=p$, $0<p<+\infty$, and

$$
\max \left\{\rho_{p}\left(A_{j}\right), j \neq s, j=0,1, \cdots, k, \rho_{p}(F)\right\}<\mu_{p}\left(A_{s}\right) \leq \rho_{p}\left(A_{s}\right)<+\infty,
$$

( $p \geq 1$ is an integer) and for some constants $0 \leq \beta<\alpha$, we have, for all $\varepsilon>0$ sufficiently small,

$$
\begin{gathered}
\left|A_{j}(z)\right| \leq \exp _{p}\left(\beta|z|^{\rho_{p}\left(A_{s}\right)-\varepsilon}\right), j \neq s, j=0,1, \cdots, k, \\
\left|A_{s}(z)\right| \geq \exp _{p}\left(\alpha|z|^{\rho_{p}\left(A_{s}\right)-\varepsilon}\right)
\end{gathered}
$$

as $z \rightarrow \infty$ for $z \in H$. Then every transcendental meromorphic solution $f$ of equation (1.4) with $\lambda_{p}\left(\frac{1}{f}\right)<\mu_{p}(f)$ satisfies $\bar{\lambda}_{p+1}(f)=\lambda_{p+1}(f)=\rho_{p+1}(f)=\rho_{p}\left(A_{s}\right)$.

Remark 1.2. Following the Lemma 2.7 of this paper, when the dominant coefficient in equation (1.3) is $A_{0}(z)$, then we obtain that every solution $f \not \equiv 0$ of equation (1.3) with $\lambda_{p}\left(\frac{1}{f}\right)<\mu_{p}(f)$ satisfies $\rho_{p}(f)=+\infty$ and $\bar{\lambda}_{p+1}(f)=\lambda_{p+1}(f)=\rho_{p+1}(f)=\rho_{p}\left(A_{0}\right)$.

Now there exists another question: How about the growth of meromorphic solutions of equation (1.4) if $\rho(F)=+\infty$ ? With regard to above question, very recently, Chen [6] investigated the growth and fixed points of solutions and their derivatives of the equation

$$
f^{\prime \prime}+A(z) f^{\prime}+B(z) f=F,
$$

where $A(z), B(z)(\not \equiv 0), F(z) \not \equiv 0$ are entire functions with $\rho(F)=+\infty$ and obtained some precise estimates of the hyper-order, the hyper-exponent of convergence of distinct zeros and the hyper-exponent of convergence of fixed points of the solutions of equation (1.5) and their 1st, 2nd derivatives.

Theorem $\mathbf{C}[6]$ Let $H$ be a set of complex numbers satisfying $\overline{\log \text { dens }}\{|z|: z \in H\}>0$, $A(z), B(z)$ be entire functions such that for some constants $\alpha \geq 0, \mu \geq 0$, we have

$$
|A(z)| \leq \exp \left\{\alpha|z|^{\mu}\right\}<|B(z)|
$$

as $z \rightarrow \infty$ for $z \in H$ and let $\rho(A) \leq \rho(B)=\mu$, and let $F(z)$ be an entire function with $\rho(F)=+\infty$. Then every solution $f$ of equation (1.5) satisfies $\rho(f)=+\infty$.

(i) If $\rho_{2}(F) \geq \mu$, then every solution $f$ of equation (1.5) satisfies $\rho_{2}(f)=\rho_{2}(F)$;

(ii) If $\rho_{2}(F)<\mu$, then every solution $f$ of equation (1.5) satisfies $\bar{\lambda}_{2}(f)=\lambda_{2}(f)=$ $\rho_{2}(f)=\mu$ with at most one exceptional solution $f_{0}$ satisfying $\rho_{2}\left(f_{0}\right)<\mu$. 
Theorem $\mathbf{D}[6]$ Let $A(z), B(z), F(z)$ and $H$ satisfy all of the hypotheses of Theorem $C(i i)$. Then every solution $f$ of equation (1.5) satisfies $\bar{\lambda}_{2}(f-z)=\bar{\lambda}_{2}\left(f^{\prime}-z\right)=\bar{\lambda}_{2}\left(f^{\prime \prime}-z\right)=$ $\rho_{2}(f)=\mu$ with at most one exceptional solution $f_{0}$ satisfying $\rho_{2}\left(f_{0}\right)<\mu$.

Recently, El Farissi in [8] investigated the relationship between solutions and their derivatives of the homogeneous differential equation (1.1) for $k \geq 2$ and small functions, where $A_{j}(j=0,1, \cdots, k-1)$ are meromorphic functions of finite iterated $p$-order and obtained the following result.

Theorem $\mathbf{E}[8]$ Let $A_{j}(z)(j=0,1, \cdots, k-1)$ be meromorphic functions of finite iterated $p$ order. Suppose that all solutions of the equation (1.1) are of infinite $p$-order and $\rho_{p+1}(f)=$ $\rho$. If $\varphi(z)(\not \equiv 0)$ is a meromorphic function satisfying $i(\varphi)<p+1$ or $\rho_{p+1}(\varphi)<\rho$, then every meromorphic solution $f$ of equation (1.1) satisfies $i_{\bar{\lambda}}\left(f^{(i)}-\varphi\right)=i_{\lambda}\left(f^{(i)}-\varphi\right)=$ $i(f)=p+1$ and $\bar{\lambda}_{p+1}\left(f^{(i)}-\varphi\right)=\lambda_{p+1}\left(f^{(i)}-\varphi\right)=\rho_{p+1}(f)=\rho,(i=0,1, \cdots)$.

In the present paper, we continue to study the oscillation problem of solutions and their derivatives, we improve and extend Theorem $\mathrm{C}$, Theorem $\mathrm{D}$ and Theorem $\mathrm{E}$ for equations of the form (1.4) by using the concept of the iterated order. We obtain the following results.

Theorem 1.1 Let $H$ be a set of complex numbers satisfying $\overline{\log \text { dens }}\{|z|: z \in H\}>0$ $\left(\right.$ or $\left.m_{l}(\{|z|: z \in H\})=+\infty\right)$ and let $A_{j}(z)(j=0,1, \cdots, k), A_{k}(z) \not \equiv 0, F(z)$ be entire functions such that $\rho_{p}(F)=+\infty$. Suppose that $i\left(A_{0}\right)=p, 0<p<+\infty$, and

$$
\max \left\{\rho_{p}\left(A_{j}\right): j=1, \cdots, k\right\}<\mu_{p}\left(A_{0}\right) \leq \rho_{p}\left(A_{0}\right)<+\infty,
$$

( $p \geq 1$ is an integer) and for some constants $0 \leq \beta<\alpha$, we have,

$$
\begin{gathered}
\left|A_{j}(z)\right| \leq \exp _{p}\left(\beta|z|^{\rho_{p}\left(A_{0}\right)}\right), j=1, \cdots, k, \\
\left|A_{0}(z)\right| \geq \exp _{p}\left(\alpha|z|^{\rho_{p}\left(A_{0}\right)}\right),
\end{gathered}
$$

as $z \rightarrow \infty$ for $z \in H$. Then every meromorphic solution $f$ of equation (1.4) with $\lambda_{p}\left(\frac{1}{f}\right)<$ $\mu_{p}(f)$ satisfies $\rho_{p}(f)=+\infty$.

(i) If $\rho_{p+1}(F) \geq \rho_{p}\left(A_{0}\right)$, then every meromorphic solution $f$ of equation (1.4) with $\lambda_{p}\left(\frac{1}{f}\right)<$ $\mu_{p}(f)$ satisfies $\rho_{p+1}(f)=\rho_{p+1}(F)$;

(ii) If $\rho_{p+1}(F)<\rho_{p}\left(A_{0}\right)$, then every meromorphic solution $f$ of equation (1.4) with $\lambda_{p}\left(\frac{1}{f}\right)<$ $\mu_{p}(f)$ satisfies $\bar{\lambda}_{p+1}(f)=\lambda_{p+1}(f)=\rho_{p+1}(f)=\rho_{p}\left(A_{0}\right)$ with at most one exceptional solution $f_{0}$ satisfying $\rho_{p+1}\left(f_{0}\right)<\rho_{p}\left(A_{0}\right)$. 
Example 1.1 Consider the differential equation

$$
\begin{gathered}
z f^{\prime \prime \prime}+3 f^{\prime \prime}+z e^{-2 i z} f=\left(\cos 2 z-\left(\frac{3}{2}+i\right) \sin 2 z\right) \cosh (\sin z) \\
-\left(\sin ^{2} z \cos z\right) \sinh (\sin z)
\end{gathered}
$$

In this equation, we have $A_{0}(z)=z e^{-2 i z}, A_{1}(z)=0, A_{2}(z)=3, A_{3}(z)=z$,

$$
F(z)=\left(\cos 2 z-\left(\frac{3}{2}+i\right) \sin 2 z\right) \cosh (\sin z)-\left(\sin ^{2} z \cos z\right) \sinh (\sin z)
$$

and

$$
\begin{gathered}
\mu\left(A_{0}\right)=\rho\left(A_{0}\right)=1, \rho\left(A_{1}\right)=0, \rho\left(A_{2}\right)=0, \rho\left(A_{3}\right)=0, \\
\rho(F)=+\infty, \rho_{2}(F)=1 .
\end{gathered}
$$

Set

$$
H=\left\{z \in \mathbb{C}: z=r e^{i \theta}, r \in\left[6,+\infty\left[, \frac{\pi}{4} \leq \theta \leq \frac{\pi}{3}\right\}\right.\right.
$$

Then

$$
\begin{gathered}
\left|A_{0}(z)\right|=\left|z e^{-2 i z}\right|=r e^{2 r \sin \theta} \geq e^{r \sqrt{2}},\left|A_{1}(z)\right|=0<\exp \{r\}, \\
\left|A_{2}(z)\right|=3 \leq \exp \{r\},\left|A_{3}(z)\right|=r \leq \exp \{r\}
\end{gathered}
$$

as $z \rightarrow \infty$ for $z \in H$. It is easy to see that the conditions (1.6), (1.7) and (1.8) of Theorem 1.1 are satisfied with $\alpha=\sqrt{2}$ and $\beta=1$. Since $\rho_{2}(F)=\rho\left(A_{0}\right)=1$, then by Theorem 1.1 (i), every meromorphic solution $f$ of equation $(*)$ with $\lambda\left(\frac{1}{f}\right)<\mu(f)$ satisfies $\rho_{2}(f)=$ $\rho(F)$. We see for example that the function $f(z)=\frac{\cosh (\sin z)}{z}$ with $\lambda\left(\frac{1}{f}\right)=0<\mu(f)=$ $\rho(f)=+\infty$ satisfies equation $(*)$ and we have $\rho_{2}(F)=\stackrel{z}{\rho_{2}}(f)=1$.

Example 1.2 Consider the differential equation

$$
\begin{gathered}
z^{2} f^{\prime \prime \prime}-6 f^{\prime}+z^{2} e^{-2 i z^{2}} f=(3-z \sin 2 z) \sin z \sinh (\sin z) \\
+\left(z e^{-2 i z^{2}}-3 \cos z(z \sin z+\cos z)\right) \cosh (\sin z)
\end{gathered}
$$

In this equation, we have $A_{0}(z)=z^{2} e^{-2 i z^{2}}, A_{1}(z)=-6, A_{2}(z)=0, A_{3}(z)=z^{2}$,

$$
\begin{gathered}
F(z)=(3-z \sin 2 z) \sin z \sinh (\sin z) \\
+\left(z e^{-2 i z^{2}}-3 \cos z(z \sin z+\cos z)\right) \cosh (\sin z)
\end{gathered}
$$


and

$$
\begin{gathered}
\mu\left(A_{0}\right)=\rho\left(A_{0}\right)=2, \rho\left(A_{1}\right)=0, \rho\left(A_{2}\right)=0, \rho\left(A_{3}\right)=0, \\
\rho(F)=+\infty, \rho_{2}(F)=1 .
\end{gathered}
$$

Let

$$
H=\left\{z \in \mathbb{C}: z=r e^{i \theta}, r \in\left[6,+\infty\left[, \frac{\pi}{8} \leq \theta \leq \frac{\pi}{6}\right\}\right.\right.
$$

Then

$$
\begin{gathered}
\left|A_{0}(z)\right|=\left|z^{2} e^{-2 i z^{2}}\right|=r^{2} e^{2 r^{2} \sin 2 \theta} \geq e^{r^{2} \sqrt{2}},\left|A_{1}(z)\right|=6<\exp \left\{r^{2}\right\}, \\
\left|A_{2}(z)\right|=0<\exp \left\{r^{2}\right\},\left|A_{3}(z)\right|=r^{2} \leq \exp \left\{r^{2}\right\}
\end{gathered}
$$

as $z \rightarrow \infty$ for $z \in H$. It is easy to see that the conditions (1.6), (1.7) and (1.8) of Theorem 1.1 are satisfied with $\alpha=\sqrt{2}$ and $\beta=1$. Since $\rho_{2}(F)=1<2=\rho\left(A_{0}\right)$, then by Theorem 1.1 (ii), every meromorphic solution $f$ of equation $(* *)$ with $\lambda\left(\frac{1}{f}\right)<\mu(f)$ satisfies $\bar{\lambda}_{2}(f)=$ $\lambda_{2}(f)=\rho_{2}(f)=\rho\left(A_{0}\right)$ with at most one exceptional solution. We see for example that the function $f_{0}(z)=\frac{\cosh (\sin z)}{z}$ with $\lambda\left(\frac{1}{f_{0}}\right)=0<\mu\left(f_{0}\right)=\rho\left(f_{0}\right)=+\infty$ is an exceptional solution which satisfies equation $(* *)$ and we have $\rho_{2}\left(f_{0}\right)=1<\rho\left(A_{0}\right)=2$.

Let $A_{0}(z) \not \equiv 0, A_{1}(z), \cdots, A_{k}(z) \not \equiv 0$ and $F(z) \not \equiv 0$ be entire functions. We define the following sequence of functions.

$$
\left\{\begin{array}{c}
A_{j}^{0}(z)=A_{j}(z), j=0,1, \cdots, k \\
A_{j}^{i}(z)=\left(A_{j+1}^{i-1}(z)\right)^{\prime}+A_{j}^{i-1}(z)-A_{j+1}^{i-1}(z) \frac{\left(A_{0}^{i-1}(z)\right)^{\prime}}{A_{0}^{i-1}(z)} \\
i=1,2, \cdots ; j=0, \cdots, k-1, \\
A_{k}^{i}(z)=A_{k}^{i-1}(z)=\cdots=A_{k}^{0}(z)=A_{k}(z) \text { for all } i \in \mathbb{N}, \\
F^{0}(z)=F(z), F^{i}(z)=\left(F^{i-1}(z)\right)^{\prime}-F^{i-1}(z) \frac{\left(A_{0}^{i-1}(z)\right)^{\prime}}{A_{0}^{i-1}(z)}, i=1,2, \cdots .
\end{array}\right.
$$

Corollary 1.1 Let $A_{j}(z)(j=0,1, \cdots, k), F(z), H$ satisfy all of the hypotheses of Theorem 1.1. Suppose that $f$ is a solution of (1.4) with $\lambda_{p}\left(\frac{1}{f}\right)<\mu_{p}(f)$ and let $A_{j}^{i}(z)$, $F^{i}(z),(j=0,1, \cdots, k), i \in \mathbb{N}$ be defined as in (1.9). Then every meromorphic solution $g$ of the equation

$$
A_{k}^{i}(z) g^{(k)}+A_{k-1}^{i}(z) g^{(k-1)}+\cdots+A_{1}^{i}(z) g^{\prime}+A_{0}^{i}(z) g=F^{i}(z)
$$

satisfies $i(g)=p+1, \rho_{p}(g)=+\infty$ and $\rho_{p+1}(g)=\rho_{p}\left(A_{0}\right)$ with at most one exceptional solution $g_{0}$ satisfying $\rho_{p+1}\left(g_{0}\right)<\rho_{p}\left(A_{0}\right)$. 
In the following theorem, we study the stability of the exponent of convergence of the sequence of zeros (resp. distinct zeros) of solutions for higher order differential equation (1.4) with their derivatives.

Theorem 1.2 Let $A_{j}(z)(j=0,1, \cdots, k), F(z), H$ satisfy all of the hypotheses of Theorem 1.1 (ii), and let $\varphi(z)(\not \equiv 0)$ be a meromorphic function satisfying $i(\varphi)<p$ or $\rho_{p+1}(\varphi)<$ $\rho_{p}\left(A_{0}\right)$. Then every meromorphic solution $f$ with $\lambda_{p}\left(\frac{1}{f}\right)<\mu_{p}(f)$ of equation (1.4) satisfies $i_{\bar{\lambda}}\left(f^{(i)}-\varphi\right)=i_{\lambda}\left(f^{(i)}-\varphi\right)=p+1$ and $\bar{\lambda}_{p+1}\left(f^{(i)}-\varphi\right)=\lambda_{p+1}\left(f^{(i)}-\varphi\right)=\rho_{p+1}(f)=$ $\rho_{p}\left(A_{0}\right),(i=0,1, \cdots)$ with at most one exceptional solution $f_{0}$ satisfying $\rho_{p+1}\left(f_{0}\right)<\rho_{p}\left(A_{0}\right)$.

Example 1.3 Consider the differential equation

$$
z^{2} f^{\prime \prime \prime}+2 z f^{\prime \prime}-2 f^{\prime}+z e^{-4 i z^{3}} f=e^{e^{z}}\left[(z-1) e^{z}+(3 z-1) e^{2 z}+z e^{3 z}+e^{-4 i z^{3}}\right]
$$

In this equation, we have $A_{0}(z)=z e^{-4 i z^{3}}, A_{1}(z)=-2, A_{2}(z)=2 z, A_{3}(z)=z^{2}$,

$$
F(z)=e^{e^{z}}\left[(z-1) e^{z}+(3 z-1) e^{2 z}+z e^{3 z}+e^{-4 i z^{3}}\right]
$$

and

$$
\begin{gathered}
\mu\left(A_{0}\right)=\rho\left(A_{0}\right)=3, \rho\left(A_{1}\right)=0, \rho\left(A_{2}\right)=0, \rho\left(A_{3}\right)=0, \\
\rho(F)=+\infty, \rho_{2}(F)=1 .
\end{gathered}
$$

Let

$$
H=\left\{z \in \mathbb{C}: z=r e^{i \theta}, r \in\left[2,+\infty\left[, \frac{\pi}{12} \leq \theta \leq \frac{\pi}{9}\right\}\right.\right.
$$

Then

$$
\begin{gathered}
\left|A_{0}(z)\right|=\left|z e^{-4 i z^{3}}\right|=r e^{4 r^{3} \sin 3 \theta} \geq e^{2 \sqrt{2} r^{3}} \\
\left|A_{1}(z)\right|=2 \leq \exp \left\{r^{3}\right\}, \\
\left|A_{2}(z)\right|=2 r \leq \exp \left\{r^{3}\right\},\left|A_{3}(z)\right|=r^{2} \leq \exp \left\{r^{3}\right\}
\end{gathered}
$$

as $z \rightarrow \infty$ for $z \in H$. It is easy to see that the conditions (1.6), (1.7) and (1.8) of Theorem 1.1 are satisfied with $\alpha=2 \sqrt{2}$ and $\beta=1$. Since $\rho_{2}(F)=1<3=\rho\left(A_{0}\right)$, then by Theorem 1.2, every meromorphic solution $f$ with $\lambda\left(\frac{1}{f}\right)<\mu(f)$ of equation $(* * *)$ satisfies $i_{\bar{\lambda}}\left(f^{(i)}-\varphi\right)=i_{\lambda}\left(f^{(i)}-\varphi\right)=2$ and $\bar{\lambda}_{2}\left(f^{(i)}-\varphi\right)=\lambda_{2}\left(f^{(i)}-\varphi\right)=\rho_{2}(f)=\rho\left(A_{0}\right)=3$, $(i=0,1, \cdots)$ with at most one exceptional solution $f_{0}$ satisfying $\rho_{2}\left(f_{0}\right)<\rho\left(A_{0}\right)$, where $\varphi(z)(\not \equiv 0)$ is a meromorphic function satisfying $\rho(\varphi)<+\infty$ or $\rho_{2}(\varphi)<\rho\left(A_{0}\right)$. We see for example that the function $f_{0}(z)=\frac{e^{e^{z}}}{z}$ with $\lambda\left(\frac{1}{f_{0}}\right)=0<\mu\left(f_{0}\right)=\rho\left(f_{0}\right)=+\infty$ is an exceptional solution which satisfies equation $(* * *)$ such that $\rho_{2}\left(f_{0}\right)=1<\rho\left(A_{0}\right)=3$. 
When $\varphi(z)=z$ in Theorem 1.2, we obtain the following corollary which improve and generalize Theorem $\mathrm{D}$.

Corollary 1.2 Let $A_{j}(z)(j=0,1, \cdots, k), F(z), H$ satisfy all of the hypothesis of Theorem 1.1 (ii). Then every meromorphic solution $f$ with $\lambda_{p}\left(\frac{1}{f}\right)<\mu_{p}(f)$ of equation (1.4) satisfies $i_{\bar{\lambda}}\left(f^{(i)}\right)=i_{\lambda}\left(f^{(i)}\right)=p+1$ and $\bar{\lambda}_{p+1}\left(f^{(i)}-z\right)=\lambda_{p+1}\left(f^{(i)}-z\right)=\rho_{p+1}(f)=\rho_{p}\left(A_{0}\right)$, $(i=0,1, \cdots)$ with at most one exceptional solution $f_{0}$ satisfying $\rho_{p+1}\left(f_{0}\right)<\rho_{p}\left(A_{0}\right)$.

\section{Preliminary lemmas}

We need the following lemmas to prove our results.

Lemma 2.1 [11] Let $f$ be a transcendental meromorphic function in the plane, and let $\alpha>1$ be a given constant. Then there exist a set $E_{1} \subset(1,+\infty)$ that has a finite logarithmic measure, and a constant $B>0$ depending only on $\mu$ and $(m, n)(m, n \in\{0,1, \cdots, k\}) m<n$ such that for all $z$ with $|z|=r \notin[0,1] \cup E_{1}$, we have

$$
\left|\frac{f^{(n)}(z)}{f^{(m)}(z)}\right| \leq B\left(\frac{T(\alpha r, f)}{r}\left(\log ^{\alpha} r\right) \log T(\alpha r, f)\right)^{n-m} .
$$

By using similar proof of Lemma 3.5 in [19], we easily obtain the following lemma when $\rho_{p}(f)=\rho_{p}(g)=+\infty$.

Lemma 2.2 Let $f(z)=\frac{g(z)}{d(z)}$ be a meromorphic function, where $g(z), d(z)$ are entire functions of finite iterated order satisfying $\mu_{p}(g)=\mu_{p}(f)=\mu \leq \rho_{p}(f)=\rho_{p}(g) \leq+\infty, 0<$ $p<+\infty, i(d)<p$ or $\rho_{p}(d)<\mu$. Let $z$ be a point with $|z|=r$ at which $|g(z)|=M(r, g)$ and let $v_{g}(r)$ denote the central index of $g$. Then the estimate

$$
\frac{f^{(n)}(z)}{f(z)}=\left(\frac{v_{g}(r)}{z}\right)^{n}(1+o(1)), n \geq 1
$$

holds for all $|z|=r$ outside a set $E_{2}$ of $r$ of finite logarithmic measure.

Lemma 2.3 [5] Let $g(z)$ be an entire function of finite iterated order satisfying $i(g)=p+1$, $\rho_{p+1}(g)=\rho, i_{\mu}(g)=q+1, \mu_{q+1}(g)=\mu, 0<p, q<+\infty$, and let $v_{g}(r)$ be the central index of $g$. Then we have

$$
\limsup _{r \rightarrow+\infty} \frac{\log _{p+1} v_{g}(r)}{\log r}=\rho, \liminf _{r \rightarrow+\infty} \frac{\log _{q+1} v_{g}(r)}{\log r}=\mu .
$$


Lemma $2.4[12]$ Let $\varphi:[0,+\infty) \rightarrow \mathbb{R}$ and $\psi:[0,+\infty) \rightarrow \mathbb{R}$ be monotone nondecreasing functions such that $\varphi(r) \leq \psi(r)$ for all $r \notin\left(E_{3} \cup[0,1]\right)$, where $E_{3}$ is a set of finite logarithmic measure. Let $\alpha>1$ be a given constant. Then there exists an $r_{1}=r_{1}(\alpha)>0$ such that $\varphi(r) \leq \psi(\alpha r)$ for all $r>r_{1}$.

Lemma 2.5 [9] Let $p \geq 1$ be an integer, and let $f(z)$ be an entire function such that $i(f)=p, \rho_{p}(f)=\rho<+\infty$. Then, there exists a set $E_{4} \subset(1,+\infty)$ of $r$ of finite linear measure such that for any given $\varepsilon>0$, we have

$$
\exp \left\{-\exp _{p-1}\left\{r^{\rho+\varepsilon}\right\}\right\} \leq|f(z)| \leq \exp _{p}\left\{r^{\rho+\varepsilon}\right\} \quad\left(r \notin E_{4}\right) .
$$

Lemma 2.6[18] Let $f(z)=\frac{g(z)}{d(z)}$ be a meromorphic function, where $g(z), d(z)$ are entire functions. If $0 \leq \rho_{p}(d)<\mu_{p}(f)$, then $\mu_{p}(g)=\mu_{p}(f)$ and $\rho_{p}(g)=\rho_{p}(f)$. Moreover, if $\rho_{p}(f)=+\infty$, then $\rho_{p+1}(g)=\rho_{p+1}(f)$.

Lemma 2.7 Let $H$ be a set of complex numbers satisfying $\overline{\log d e n s}\{|z|: z \in H\}>0\left(\right.$ or $\left.m_{l}(\{|z|: z \in H\})=+\infty\right)$ and let $A_{j}(z)(j=0,1, \cdots, k)$ be entire functions such that $A_{k}(z) \not \equiv 0$. Suppose that $i\left(A_{0}\right)=$ $p, 0<p<+\infty$, and

$$
\max \left\{\rho_{p}\left(A_{j}\right): j=1, \cdots, k\right\}<\mu_{p}\left(A_{0}\right) \leq \rho_{p}\left(A_{0}\right)<+\infty,
$$

( $p \geq 1$ is an integer) and for some constants $0 \leq \beta<\alpha$, we have,

$$
\begin{gathered}
\left|A_{j}(z)\right| \leq \exp _{p}\left(\beta|z|^{\rho_{p}\left(A_{0}\right)}\right), j=1, \cdots, k, \\
\left|A_{0}(z)\right| \geq \exp _{p}\left(\alpha|z|^{\rho_{p}\left(A_{0}\right)}\right)
\end{gathered}
$$

as $z \rightarrow \infty$ for $z \in H$. Then every meromorphic solution $f \not \equiv 0$ of equation (1.3) with $\lambda_{p}\left(\frac{1}{f}\right)<\mu_{p}(f)$ satisfies $\mu_{p}(f)=\rho_{p}(f)=+\infty$ and $\rho_{p+1}(f)=\rho_{p}\left(A_{0}\right)$.

Proof. Let $f \not \equiv 0$ be a meromorphic solution of equation (1.3) with $\lambda_{p}\left(\frac{1}{f}\right)<\mu_{p}(f)$. By (1.3), we have

$$
\left|A_{0}(z)\right| \leq \sum_{j=1}^{k}\left|A_{j}(z)\right|\left|\frac{f^{(j)}}{f}\right| .
$$

Using Lemma 2.1, there exists a set $E_{1} \subset(1,+\infty)$ with $m_{l}\left(E_{1}\right)<+\infty$ and a constant $B>0$, such that for all $z$ satisfying $|z|=r \notin E_{1} \cup[0,1]$

$$
\left|\frac{f^{(j)}(z)}{f(z)}\right| \leq B[T(2 r, f)]^{k+1}, j=1,2, \cdots, k .
$$


By the hypotheses of Lemma 2.7, there exists a set $H$ with $\overline{\log d e n s}\{|z|: z \in H\}>0$ (or $m_{l}(\{|z|: z \in H\})=+\infty$ ) such that for all $z \in H$ as $z \rightarrow \infty$, we have

$$
\begin{gathered}
\left|A_{j}(z)\right| \leq \exp _{p}\left(\beta|z|^{\rho_{p}\left(A_{0}\right)}\right), j=1, \cdots, k, \\
\left|A_{0}(z)\right| \geq \exp _{p}\left(\alpha|z|^{\rho_{p}\left(A_{0}\right)}\right) .
\end{gathered}
$$

Set $H_{1}=\{|z|: z \in H\} \backslash\left(E_{1} \cup[0,1]\right)$, so $m_{l}\left(H_{1}\right)=+\infty$. By substituting (2.2), (2.3) and (2.4) into (2.1), for all $z$ satisfying $|z|=r \in H_{1}$, we have

$$
\exp _{p}\left(\alpha|z|^{\rho_{p}\left(A_{0}\right)}\right) \leq k B(T(2 r, f))^{k+1} \exp _{p}\left(\beta|z|^{\rho_{p}\left(A_{0}\right)}\right)
$$

since $0 \leq \beta<\alpha$, then we obtain

$$
\exp \left((1-o(1)) \exp _{p-1}\left(\alpha|z|^{\rho_{p}\left(A_{0}\right)}\right)\right) \leq k B(T(2 r, f))^{k+1} .
$$

It follows from (2.5) that

$$
\mu_{p}(f)=\rho_{p}(f)=+\infty
$$

and

$$
\rho_{p}\left(A_{0}\right) \leq \rho_{p+1}(f) .
$$

On the other hand, by the hypotheses of Lemma 2.7, for sufficiently large $r$, we have

$$
\left|A_{j}(z)\right| \leq \exp _{p}\left(r^{\rho_{p}\left(A_{0}\right)+\varepsilon}\right), j=0,1, \cdots, k
$$

By Lemma 2.5, for any given $\varepsilon>0$, there exists a set $E_{4} \subset(1,+\infty)$ of finite linear measure, such that for all $z$ satisfying $|z|=r \notin E_{4}$, we obtain

$$
\left|A_{k}(z)\right| \geq \exp \left\{-\exp _{p-1}\left(r^{\rho_{p}\left(A_{k}\right)+\varepsilon}\right)\right\} \geq \exp \left\{-\exp _{p-1}\left(r^{\rho_{p}\left(A_{0}\right)+\varepsilon}\right)\right\} .
$$

It follows by (1.3) that

$$
\left|\frac{f^{(k)}(z)}{f(z)}\right| \leq \frac{1}{\left|A_{k}(z)\right|}\left(\sum_{j=1}^{k-1}\left|A_{j}(z)\right| \frac{f^{(j)}(z)}{f(z)}|+| A_{0}(z) \mid\right) .
$$

Since $\lambda_{p}\left(\frac{1}{f}\right)<\mu_{p}(f)$, then by Hadamard factorization theorem, we can write $f$ as $f(z)=$ $\frac{g(z)}{d(z)}$, where $g(z)$ and $d(z)$ are entire functions of finite iterated order satisfying $\mu_{p}(g)=$ $\mu_{p}(f) \leq \rho_{p}(f)=\rho_{p}(g) \leq+\infty, 0<p<+\infty, i(d)<p$ or $\rho_{p}(d)=\lambda_{p}(d)=\lambda_{p}\left(\frac{1}{f}\right)<$ 
$\mu_{p}(f)$. By Lemma 2.2, there exists a set $E_{2} \subset(1,+\infty)$ of finite logarithmic measure such that for all $z$ satisfying $|z|=r \notin E_{2} \cup[0,1]$ at which $|g(z)|=M(r, g)$ we have

$$
\frac{f^{(j)}(z)}{f(z)}=\left(\frac{v_{g}(r)}{z}\right)^{j}(1+o(1)), j=1, \cdots, k .
$$

By substituting (2.6), (2.7) and (2.9) into (2.8), we obtain

$$
\begin{gathered}
\left|\frac{v_{g}(r)}{z}\right|^{k}|1+o(1)| \\
\leq \frac{1}{\exp \left\{-\exp _{p-1}\left(r^{\rho_{p}\left(A_{0}\right)+\varepsilon}\right)\right\}}\left\{\sum_{j=1}^{k-1}\left|\frac{v_{g}(r)}{z}\right|^{j}|1+o(1)|+1\right\} \exp _{p}\left(r^{\rho_{p}\left(A_{0}\right)+\varepsilon}\right) \\
=\left\{\sum_{j=1}^{k-1}\left|\frac{v_{g}(r)}{z}\right|^{j}|1+o(1)|+1\right\} \exp \left\{2 \exp _{p-1}\left(r^{\rho_{p}\left(A_{0}\right)+\varepsilon}\right)\right\} .
\end{gathered}
$$

Hence

$$
\left|v_{g}(r)\right||1+o(1)| \leq k r^{k}|1+o(1)| \exp \left\{2 \exp _{p-1}\left(r^{\rho_{p}\left(A_{0}\right)+\varepsilon}\right)\right\},
$$

for all $z$ satisfying $|z|=r \notin\left([0,1] \cup E_{2} \cup E_{4}\right)$ and $|g(z)|=M(r, g), r \rightarrow+\infty$. By (2.10) and Lemma 2.4, we get

$$
\limsup _{r \rightarrow+\infty} \frac{\log _{p+1} v_{g}(r)}{\log r} \leq \rho_{p}\left(A_{0}\right)+\varepsilon
$$

Since $\varepsilon>0$ is arbitrary, then by (2.11) and Lemma 2.3, we obtain

$$
\rho_{p+1}(g) \leq \rho_{p}\left(A_{0}\right) .
$$

Because $\rho_{p}(d)<\mu_{p}(f)$, so by Lemma 2.6 we have $\rho_{p+1}(g)=\rho_{p+1}(f)$. This and the fact that $\rho_{p}\left(A_{0}\right) \leq \rho_{p+1}(f)$ yield $i(f)=p+1$ and $\rho_{p+1}(f)=\rho_{p}\left(A_{0}\right)$. Thus, Lemma 2.7 is proved.

The proofs of the following lemma is essentially the same as in the corresponding results for the usual order. For details, see Chapter 2 of the book by Goldberg-Ostrovskii [10]. So, we omit the proofs.

Lemma 2.8 Let $f$ and $g$ be meromorphic functions with $i(f)=i(g)=p+1$. Then

$$
\begin{gathered}
\rho_{p+1}(f+g) \leq \max \left(\rho_{p+1}(f), \rho_{p+1}(g)\right), \\
\rho_{p+1}(f g) \leq \max \left(\rho_{p+1}(f), \rho_{p+1}(g)\right) .
\end{gathered}
$$


Lemma 2.9 [13] Let $f$ be a meromorphic function and let $k \in \mathbb{N}$. Then

$$
m\left(r, \frac{f^{(k)}}{f}\right)=S(r, f)
$$

where $S(r, f)=O(\log T(r, f)+\log r)$, possibly outside a set $E_{5} \subset(0,+\infty)$ with a finite linear measure. If $f$ is a finite order of growth, then

$$
m\left(r, \frac{f^{(k)}}{f}\right)=O(\log r) .
$$

Lemma 2.10 Let $A_{j}(z)(j=0,1, \cdots, k), F(z)(\not \equiv 0)$ be meromorphic functions and let $f(z)$ be a meromorphic solution of (1.4) with infinite $p$-order such that $i(f)=p+1(0<p<+\infty)$. If either

$$
b=\max \left\{\rho_{p+1}(F), \rho_{p+1}\left(A_{j}\right)(j=0,1, \cdots, k)\right\}<\rho_{p+1}(f)
$$

or

$$
\max \left\{i(F), i\left(A_{j}\right)(j=0,1, \cdots, k)\right\}<p+1,
$$

then $i_{\bar{\lambda}}(f)=i_{\lambda}(f)=i(f)=p+1, \bar{\lambda}_{p+1}(f)=\lambda_{p+1}(f)=\rho_{p+1}(f)$.

Proof. We assume $f(z)$ is a meromorphic solution of (1.4) with infinite $p$-order. By (1.4), it is easy to see that if $f$ has a zero at $z_{0}$ of order $\alpha>k$, and $A_{0}, A_{1}, \cdots, A_{k}$ are all analytic at $z_{0}$, then $F$ must have a zero at $z_{0}$ of order at least $\alpha-k$. Hence,

$$
n\left(r, \frac{1}{f}\right) \leq k \bar{n}\left(r, \frac{1}{f}\right)+n\left(r, \frac{1}{F}\right)+\sum_{j=0}^{k} n\left(r, A_{j}\right)
$$

and

$$
N\left(r, \frac{1}{f}\right) \leq k \bar{N}\left(r, \frac{1}{f}\right)+N\left(r, \frac{1}{F}\right)+\sum_{j=0}^{k} N\left(r, A_{j}\right) .
$$

Now (1.4) can be rewritten as

$$
\frac{1}{f}=\frac{1}{F}\left(A_{k} \frac{f^{(k)}}{f}+A_{k-1}(z) \frac{f^{(k-1)}}{f}+\cdots+A_{1}(z) \frac{f^{\prime}}{f}+A_{0}(z)\right) .
$$

By Lemma 2.9 and (2.13), for $|z|=r$ outside a set $E_{5}$ of finite linear measure, we have

$$
m\left(r, \frac{1}{f}\right) \leq m\left(r, \frac{1}{F}\right)+\sum_{j=1}^{k} m\left(r, \frac{f^{(j)}}{f}\right)+\sum_{j=0}^{k} m\left(r, A_{j}\right)
$$




$$
\leq m\left(r, \frac{1}{F}\right)+\sum_{j=0}^{k} m\left(r, A_{j}\right)+O(\log r T(r, f)) .
$$

Therefore, by (2.12) and (2.14), we obtain

$$
\begin{gathered}
T(r, f)=T\left(r, \frac{1}{f}\right)+O(1) \\
\leq T(r, F)+\sum_{j=0}^{k} T\left(r, A_{j}\right)+k \bar{N}\left(r, \frac{1}{f}\right)+O(\log (r T(r, f))) .
\end{gathered}
$$

for all sufficiently large $r \notin E_{5}$. For sufficiently large $r$, we have

$$
O(\log (r T(r, f))) \leq \frac{1}{2} T(r, f) .
$$

We assume first that

$$
b=\max \left\{\rho_{p+1}(F), \rho_{p+1}\left(A_{j}\right)(j=0,1, \cdots, k)\right\}<\rho_{p+1}(f) .
$$

By using the definition of the iterated order, for any given $\varepsilon\left(0<2 \varepsilon<\rho_{p+1}(f)-b\right)$, and for sufficiently large $r$, we have

$$
\begin{gathered}
T(r, F) \leq \exp _{p}\left\{r^{b+\varepsilon}\right\}=o(1) \exp _{p}\left\{r^{\rho_{p+1}(f)-\varepsilon}\right\}, \\
T\left(r, A_{j}\right) \leq \exp _{p}\left\{r^{b+\varepsilon}\right\}=o(1) \exp _{p}\left\{r^{\rho_{p+1}(f)-\varepsilon}\right\}, j=0,1, \cdots, k .
\end{gathered}
$$

By $(2.15),(2.16),(2.17)$ and $(2.18)$, for $r \notin E_{5}$ sufficiently large, we obtain

$$
T(r, f) \leq 2 k \bar{N}\left(r, \frac{1}{f}\right)+o(1) \exp _{p}\left\{r^{\rho_{p+1}(f)-\varepsilon}\right\} .
$$

Hence for any $f$, by (2.19), we have

$$
\rho_{p+1}(f) \leq \bar{\lambda}_{p+1}(f)
$$

then

$$
\rho_{p+1}(f) \leq \bar{\lambda}_{p+1}(f) \leq \lambda_{p+1}(f) .
$$

Since by definition, we have $\bar{\lambda}_{p+1}(f) \leq \lambda_{p+1}(f) \leq \rho_{p+1}(f)$, therefore

$$
\rho_{p+1}(f)=\bar{\lambda}_{p+1}(f)=\lambda_{p+1}(f) .
$$


Assume now, that $\max \left\{i(F), i\left(A_{j}\right)(j=0,1, \cdots, k)\right\}<p+1$, and hence $d=\max \left\{\rho_{p}(F), \rho_{p}\left(A_{j}\right)(j=0,1, \cdots, k)\right\}$ $+\infty$. By using the definition of the iterated order, for any given $\varepsilon\left(0<2 \varepsilon<\rho_{p+1}(f)-d\right)$, and for sufficiently large $r$, we have

$$
\begin{gathered}
T(r, F) \leq \exp _{p-1}\left\{r^{d+\varepsilon}\right\}=o(1) \exp _{p-1}\left\{r^{\rho_{p+1}(f)-\varepsilon}\right\}, \\
T\left(r, A_{j}\right) \leq \exp _{p-1}\left\{r^{d+\varepsilon}\right\}=o(1) \exp _{p-1}\left\{r^{\rho_{p+1}(f)-\varepsilon}\right\}, j=0,1, \cdots, k .
\end{gathered}
$$

By (2.15), (2.16), (2.20) and (2.21), for $r \notin E_{5}$ sufficiently large, we obtain

$$
T(r, f) \leq 2 k \bar{N}\left(r, \frac{1}{f}\right)+o(1) \exp _{p-1}\left\{r^{\rho_{p+1}(f)-\varepsilon}\right\} .
$$

Hence for any $f$, by (2.22), we have

$$
\rho_{p+1}(f) \leq \bar{\lambda}_{p+1}(f)
$$

then

$$
\rho_{p+1}(f) \leq \bar{\lambda}_{p+1}(f) \leq \lambda_{p+1}(f) .
$$

Since by definition, we have $\bar{\lambda}_{p+1}(f) \leq \lambda_{p+1}(f) \leq \rho_{p+1}(f)$, therefore

$$
\bar{\lambda}_{p+1}(f)=\lambda_{p+1}(f)=\rho_{p+1}(f) .
$$

This implies that $i_{\bar{\lambda}}(f)=i_{\lambda}(f)=i(f)=p+1$. Thus, Lemma 2.10 is proved.

Lemma 2.11 Suppose $f(z)$ and $g(z)$ are two nonconstant meromorphic function in the complex plane. If $\rho_{p}(f)=+\infty$ and $\rho_{p}(g)<+\infty$, then $\rho_{p}(f g)=+\infty$.

Proof. Suppose that $\rho_{p}(f g)<+\infty$. Then

$$
\begin{aligned}
\rho_{p}(f)= & \rho_{p}\left(f g \frac{1}{g}\right) \leq \max \left(\rho_{p}(f g), \rho_{p}\left(\frac{1}{g}\right)\right) \\
& =\max \left(\rho_{p}(f g), \rho_{p}(g)\right)<+\infty .
\end{aligned}
$$

This is a contradiction.

Lemma 2.12 [4] Let $f$ be a meromorphic function of $[p, q]$ order. If $p \geq q \geq 1$, then $\rho_{[p, q]}\left(f^{\prime}\right)=\rho_{[p, q]}(f)$. 
Lemma 2.13 Let $A_{j}(z)(j=0,1, \cdots, k), F(z) \not \equiv 0$ be entire functions such that $A_{k}(z) \not \equiv 0$, $A_{0}(z) \not \equiv 0$. Suppose that $f$ is a solution of (1.4). Then $g_{i}=f^{(i)}$ is a solution of the equation

$$
A_{k}^{i}(z) g_{i}^{(k)}+A_{k-1}^{i}(z) g_{i}^{(k-1)}+\cdots+A_{1}^{i}(z) g_{i}^{\prime}+A_{0}^{i}(z) g_{i}=F^{i}(z)
$$

where $A_{j}^{i}(z), F^{i}(z),(j=0,1, \cdots, k), i \in \mathbb{N}$ are given by (1.9).

Proof. Assume that $f$ is a solution of equation (1.4) and let $g_{i}=f^{(i)}$. We prove that $g_{i}$ is a solution of the equation (2.23). Our proof is by induction. For $i=1$, differentiating both sides of (1.4), we obtain

$$
\begin{gathered}
A_{k}(z) f^{(k+1)}+\left(A_{k}^{\prime}(z)+A_{k-1}(z)\right) f^{(k)} \\
+\cdots+\left(A_{1}^{\prime}(z)+A_{0}(z)\right) f^{\prime}+A_{0}^{\prime}(z) f=F^{\prime}(z) .
\end{gathered}
$$

By (1.4), we get

$$
f=\frac{F-\left(A_{k}(z) f^{(k)}+A_{k-1}(z) f^{(k-1)}+\cdots+A_{1}(z) f^{\prime}\right)}{A_{0}(z)} .
$$

Substituting (2.25) into (2.24), we get

$$
\begin{gathered}
A_{k}(z) f^{(k+1)}+\left(A_{k}^{\prime}(z)+A_{k-1}(z)-\frac{A_{0}^{\prime}(z)}{A_{0}(z)} A_{k}(z)\right) f^{(k)} \\
+\left(A_{k-1}^{\prime}(z)+A_{k-2}(z)-\frac{A_{0}^{\prime}(z)}{A_{0}(z)} A_{k-1}(z)\right) f^{(k-1)} \\
+\cdots+\left(A_{1}^{\prime}(z)+A_{0}(z)-\frac{A_{0}^{\prime}(z)}{A_{0}(z)} A_{1}(z)\right) f^{\prime}=F^{\prime}(z)-\frac{A_{0}^{\prime}(z)}{A_{0}(z)} F .
\end{gathered}
$$

That is

$$
A_{k}^{0}(z) g_{1}^{(k)}+A_{k-1}^{1}(z) g_{1}^{(k-1)}+A_{k-2}^{1}(z) g_{1}^{(k-2)}+\cdots+A_{0}^{1}(z) g_{1}=F^{1} .
$$

Using (1.9), (2.27) becomes

$$
A_{k}^{1}(z) g_{1}^{(k)}+A_{k-1}^{1}(z) g_{1}^{(k-1)}+A_{k-2}^{1}(z) g_{1}^{(k-2)}+\cdots+A_{0}^{1}(z) g_{1}=F^{1} .
$$

Suppose that the assertion is true for the values which are strictly smaller than a certain $i$. We suppose $g_{i-1}$ is a solution of the equation

$$
A_{k}^{i-1}(z) g_{i-1}^{(k)}+A_{k-1}^{i-1}(z) g_{i-1}^{(k-1)}+A_{k-2}^{i-1}(z) g_{i-1}^{(k-2)}+\cdots+A_{0}^{i-1}(z) g_{i-1}=F^{i-1} .
$$

Differentiating both sides of (2.28), we can write

$$
A_{k}^{i-1}(z) g_{i-1}^{(k+1)}+\left(\left(A_{k}^{i-1}(z)\right)^{\prime}+A_{k-1}^{i-1}(z)\right) g_{i-1}^{(k)}
$$




$$
+\left(\left(A_{k-1}^{i-1}(z)\right)^{\prime}+A_{k-2}^{i-1}(z)\right) g_{i-1}^{(k-1)}+\cdots+\left(A_{0}^{i-1}(z)\right)^{\prime} g_{i-1}=\left(F^{i-1}\right)^{\prime} .
$$

From (2.28), we have

$$
\begin{gathered}
g_{i-1}=\frac{F^{i-1}}{A_{0}^{i-1}(z)} \\
-\frac{A_{k}^{i-1}(z) g_{i-1}^{(k)}+A_{k-1}^{i-1}(z) g_{i-1}^{(k-1)}+A_{k-2}^{i-1}(z) g_{i-1}^{(k-2)}+\cdots+A_{1}^{i-1}(z) g_{i-1}^{\prime}}{A_{0}^{i-1}(z)} .
\end{gathered}
$$

Substituting (2.30) into (2.29), we get

$$
\begin{gathered}
A_{k}^{i-1}(z) g_{i-1}^{(k+1)}+\left(\left(A_{k}^{i-1}(z)\right)^{\prime}+A_{k-1}^{i-1}(z)-A_{k}^{i-1}(z) \frac{\left(A_{0}^{i-1}(z)\right)^{\prime}}{A_{0}^{i-1}(z)}\right) g_{i-1}^{(k)} \\
+\left(\left(A_{k-1}^{i-1}(z)\right)^{\prime}+A_{k-2}^{i-1}(z)-A_{k-1}^{i-1}(z) \frac{\left(A_{0}^{i-1}(z)\right)^{\prime}}{A_{0}^{i-1}(z)}\right) g_{i-1}^{(k-1)} \\
+\cdots+\left(\left(A_{1}^{i-1}(z)\right)^{\prime}+A_{0}^{i-1}(z)-A_{1}^{i-1}(z) \frac{\left(A_{0}^{i-1}(z)\right)^{\prime}}{A_{0}^{i-1}(z)}\right) g_{i-1}^{\prime} \\
=\left(F^{i-1}\right)^{\prime}-\frac{\left(A_{0}^{i-1}(z)\right)^{\prime}}{A_{0}^{i-1}(z)} F^{i-1}
\end{gathered}
$$

By (2.31) and (1.9), we have

$$
A_{k}^{i}(z) g_{i}^{(k)}+A_{k-1}^{i}(z) g_{i}^{(k-1)}+A_{k-2}^{i}(z) g_{i}^{(k-2)}+\cdots+A_{0}^{i}(z) g_{i}=F^{i} .
$$

Thus, Lemma 2.13 is proved.

\section{Proof of Theorems and Corollary 1.1}

Proof of Theorem 1.1. Assume $f$ is a meromorphic solution of (1.4) with $\lambda_{p}\left(\frac{1}{f}\right)<\mu_{p}(f)$. Suppose that $\rho_{p}(f)<+\infty$. Combining (1.4) and the first main theory in Nevanlinna theory, we get

$$
T(r, F) \leq \sum_{j=0}^{k} T\left(r, f^{(j)}\right)+\sum_{j=0}^{k} T\left(r, A_{j}\right)+O(1) .
$$

By using the similar way in proving $T\left(r, f^{\prime}\right) \leq 2 T(r, f)+m\left(r, \frac{f^{\prime}}{f}\right)$, see $([21]$, p. 97), for every integer $j \in[1, k]$,

$$
T\left(r, f^{(j)}\right) \leq m\left(r, \frac{f^{(j)}}{f^{(j-1)}}\right)+2 m\left(r, \frac{f^{(j-1)}}{f^{(j-2)}}\right)+\cdots+2^{j-1} m\left(r, \frac{f^{\prime}}{f}\right)+2^{j} T(r, f) .
$$


Combining (3.1) and (3.2), we get

$$
T(r, F) \leq \sum_{j=0}^{k} T\left(r, A_{j}\right)+c_{1} T(r, f)+c_{2} \sum_{j=1}^{k} m\left(r, \frac{f^{(j)}}{f^{(j-1)}}\right)+O(1),
$$

where $c_{1}>0, c_{2}>0$ are some constants. It follows from $\max \left\{\rho_{p}\left(A_{j}\right): j=1, \cdots, k\right\}<$ $\rho_{p}\left(A_{0}\right)<+\infty$ and the lemma of logarithmic derivative that

$$
\rho_{p}(F) \leq \max \left\{\rho_{p}\left(A_{0}\right), \rho_{p}(f)\right\}<+\infty
$$

which contradict the fact that

$$
\rho_{p}(F)=+\infty .
$$

Hence $\rho_{p}(f)=+\infty$. Now, we assume that $f_{1}, f_{2}, \cdots, f_{k}$ are non-trivial meromorphic solutions with $\lambda_{p}\left(\frac{1}{f_{j}}\right)<\mu_{p}\left(f_{j}\right)(j=1, \cdots, k)$ of the corresponding homogeneous equation (1.3) of (1.4). By Lemma 2.7, we get that $\rho_{p+1}\left(f_{j}\right)=\rho_{p}\left(A_{0}\right),(j=1, \cdots, k)$. By the elementary theory of differential equations, all solutions of (1.4) can be represented in the form

$$
f(z)=f_{0}(z)+B_{1} f_{1}(z)+B_{2} f_{2}(z)+\cdots+B_{k} f_{k}(z)
$$

where $B_{1}, \cdots, B_{k} \in \mathbb{C}$ and the function $f_{0}$ has the form

$$
f_{0}(z)=C_{1}(z) f_{1}(z)+C_{2}(z) f_{2}(z)+\cdots+C_{k}(z) f_{k}(z),
$$

where $C_{1}(z), \cdots, C_{k}(z)$ are suitable meromorphic functions satisfying

$$
C_{j}^{\prime}=F \cdot G_{j}\left(f_{1}, f_{2}, \cdots, f_{k}\right)\left[W\left(f_{1}, f_{2}, \cdots, f_{k}\right)\right]^{-1}, j=1, \cdots, k,
$$

where $G_{j}\left(f_{1}, f_{2}, \cdots, f_{k}\right)$ are differential polynomials in $f_{1}, f_{2}, \cdots, f_{k}$ and their derivatives with constant coefficients, and $W\left(f_{1}, f_{2}, \cdots, f_{k}\right)$ is the Wronskian of $f_{1}, f_{2}, \cdots, f_{k}$. Since the Wronskian $W\left(f_{1}, f_{2}, \cdots, f_{k}\right)$ is a differential polynomial in $f_{1}, f_{2}, \cdots, f_{k}$, by Lemma 2.8 and Lemma 2.12, we obtain

$$
\rho_{p+1}\left(W\left(f_{1}, f_{2}, \cdots, f_{k}\right)\right) \leq \max \left(\rho_{p+1}\left(f_{j}\right): j=1, \cdots, k\right)=\rho_{p}\left(A_{0}\right) .
$$

Also, we have that $G_{j}\left(f_{1}, f_{2}, \cdots, f_{k}\right)$ are differential polynomials in $f_{1}, f_{2}, \cdots, f_{k}$ and their derivatives with constant coefficients, then

$$
\rho_{p+1}\left(G_{j}\left(f_{1}, f_{2}, \cdots, f_{k}\right)\right) \leq \max \left(\rho_{p+1}\left(f_{j}\right): j=1, \cdots, k\right)=\rho_{p}\left(A_{0}\right) .
$$

By Lemma 2.12, (3.6), (3.7) and (3.8), for $j=1, \cdots, k$, we have

$$
\rho_{p+1}\left(C_{j}\right)=\rho_{p+1}\left(C_{j}^{\prime}\right) \leq \max \left(\rho_{p+1}(F), \rho_{p}\left(A_{0}\right)\right) .
$$


Hence from (3.4), (3.5) and (3.9), we obtain

$$
\begin{aligned}
\rho_{p+1}(f) \leq & \max \left(\rho_{p+1}\left(f_{j}\right), \rho_{p+1}\left(C_{j}\right), j=1, \cdots, k\right) \\
& \leq \max \left(\rho_{p+1}(F), \rho_{p}\left(A_{0}\right)\right) .
\end{aligned}
$$

(i) If $\rho_{p+1}(F) \geq \rho_{p}\left(A_{0}\right)$, then by (3.10), every meromorphic solution $f$ of equation (1.4) with $\lambda_{p}\left(\frac{1}{f}\right)<\mu_{p}(f)$ satisfies $\rho_{p+1}(f) \leq \rho_{p+1}(F)$. On the other hand, since $\max \left\{\rho_{p}\left(A_{j}\right): j=1, \cdots, k\right\}<$ $\rho_{p}\left(A_{0}\right)<+\infty$ so $\rho_{p+1}\left(A_{j}\right)=0, j=0,1, \cdots, k$, then by inequality (3.3) we obtain

$$
\rho_{p+1}(F) \leq \rho_{p+1}(f) .
$$

Hence, every meromorphic solution $f$ of equation (1.4) with $\lambda_{p}\left(\frac{1}{f}\right)<\mu_{p}(f)$ satisfies $\rho_{p+1}(f)=\rho_{p+1}(F)$.

(ii) If $\rho_{p+1}(F)<\rho_{p}\left(A_{0}\right)$, then (3.10) gives that every meromorphic solution $f$ of equation (1.4) with $\lambda_{p}\left(\frac{1}{f}\right)<\mu_{p}(f)$ satisfies $\rho_{p+1}(f) \leq \rho_{p}\left(A_{0}\right)$. On the other hand, we affirm that (1.4) can only possess at most one exceptional solution $f_{0}$ satisfying $\rho_{p+1}\left(f_{0}\right)<\rho_{p}\left(A_{0}\right)$. In fact, if there exists a second solution $f_{*}$ of $(1.4)$ that satisfies $\rho_{p+1}\left(f_{*}\right)<\rho_{p}\left(A_{0}\right)$, then by Lemma 2.8, we get $\rho_{p+1}\left(f_{0}-f_{*}\right) \leq \max \left(\rho_{p+1}\left(f_{0}\right), \rho_{p+1}\left(f_{*}\right)\right)<\rho_{p}\left(A_{0}\right)$. But $f_{0}-f_{*}$ is a solution of (1.3), this contradicts Lemma 2.7. Then, $\rho_{p+1}(f)=\rho_{p}\left(A_{0}\right)$ holds for all solutions of (1.4) with at most one exceptional solution $f_{0}$ satisfying $\rho_{p+1}\left(f_{0}\right)<\rho_{p}\left(A_{0}\right)$. Since $\rho_{p+1}(F)<\rho_{p}\left(A_{0}\right)$, then

$$
\max \left\{\rho_{p+1}(F), \rho_{p+1}\left(A_{j}\right)(j=0,1, \cdots, k)\right\}<\rho_{p}\left(A_{0}\right)=\rho_{p+1}(f) .
$$

Therefore, by Lemma 2.10, we get that

$$
\bar{\lambda}_{p+1}(f)=\lambda_{p+1}(f)=\rho_{p+1}(f)=\rho_{p}\left(A_{0}\right)
$$

holds for all meromorphic solutions $f$ of (1.4) satisfying $\lambda_{p}\left(\frac{1}{f}\right)<\mu_{p}(f)$ with at most one exceptional solution $f_{0}$ satisfying $\rho_{p+1}\left(f_{0}\right)<\rho_{p}\left(A_{0}\right)$. This complete the proof of Theorem 1.1.

Proof of Corollary 1.1 Let $\left\{f_{1}, f_{2}, \cdots, f_{k}\right\}$ be a fundamental system of meromorphic solutions satisfying $\lambda_{p}\left(\frac{1}{f_{j}}\right)<\mu_{p}\left(f_{j}\right)(j=1, \cdots, k)$ of the corresponding homogeneous equation (1.3) of (1.4). We show that $\left\{f_{1}^{(i)}, f_{2}^{(i)}, \cdots, f_{k}^{(i)}\right\}$ is a fundamental system of meromorphic solutions of the corresponding homogeneous equation

$$
A_{k}^{i}(z) g^{(k)}+A_{k-1}^{i}(z) g^{(k-1)}+\cdots+A_{1}^{i}(z) g^{\prime}+A_{0}^{i}(z) g=0
$$


of (1.10). By Lemma 2.13, it follows that $f_{1}^{(i)}, f_{2}^{(i)}, \cdots, f_{k}^{(i)}$ are solutions of $(2.23)$. Let $\alpha_{1}, \alpha_{2}, \cdots, \alpha_{k}$ be constants such that

$$
\alpha_{1} f_{1}^{(i)}+\alpha_{2} f_{2}^{(i)}+\cdots+\alpha_{k} f_{k}^{(i)}=0
$$

Then, we have

$$
\alpha_{1} f_{1}+\alpha_{2} f_{2}+\cdots+\alpha_{k} f_{k}=P(z)
$$

where $P(z)$ is a polynomial of degree less than $i$. Since $\alpha_{1} f_{1}+\alpha_{2} f_{2}+\cdots+\alpha_{k} f_{k}$ is a solution of the corresponding homogeneous equation (1.3) of (1.4), then $P$ is a solution of (1.3) and by Lemma 2.7, we conclude that $P$ is an infinite iterated $p$-order solution of (1.3), this leads to a contradiction. Therefore, $P$ is a trivial solution. We deduce that $\alpha_{1} f_{1}+\alpha_{2} f_{2}+\cdots+\alpha_{k} f_{k}=0$. Using the fact that $\left\{f_{1}, f_{2}, \cdots, f_{k}\right\}$ is a fundamental system of meromorphic solutions of (1.3), we get $\alpha_{1}=\alpha_{2}=\cdots=\alpha_{k}=0$. Now, let $g$ be a non-trivial solution of (1.10). Then, using the fact that $\left\{f_{1}^{(i)}, f_{2}^{(i)}, \cdots, f_{k}^{(i)}\right\}$ is a fundamental system of meromorphic solutions of (3.12), we claim that there exist constants $\alpha_{1}, \alpha_{2}, \cdots, \alpha_{k}$ not all equal to zero, such that

$$
g=\alpha_{1} f_{1}^{(i)}+\alpha_{2} f_{2}^{(i)}+\cdots+\alpha_{k} f_{k}^{(i)}
$$

Let

$$
h=\alpha_{1} f_{1}+\alpha_{2} f_{2}+\cdots+\alpha_{k} f_{k},
$$

be a solution of (1.4) and $h^{(i)}=g$. Then, by Lemma 2.12, we have $\rho_{p+1}(h)=\rho_{p+1}(g)$ and by the conditions of the Corollary 1.1 , we obtain $\rho_{p}(h)=\rho_{p}(g)=+\infty$ and $\rho_{p+1}(h)=$ $\rho_{p+1}(g)=\rho_{p}\left(A_{0}\right)$ with at most one exceptional solution $g_{0}$ satisfying $\rho_{p+1}\left(g_{0}\right)<\rho_{p}\left(A_{0}\right)$.

Proof of Theorem 1.2 Assume $f$ is a meromorphic solution of $(1.4)$ with $\lambda_{p}\left(\frac{1}{f}\right)<\mu_{p}(f)$. Then by Theorem 1.1 (ii), we get $i(f)=p+1, \rho_{p+1}(f)=\rho_{p}\left(A_{0}\right)$ with at most one exceptional solution $f_{0}$ satisfying $\rho_{p+1}\left(f_{0}\right)<\rho_{p}\left(A_{0}\right)$. Taking $g_{i}=f^{(i)}$, then by using Lemma 2.13, we have $g_{i}$ is a solution of (2.23) and by Corollary 1.1, we obtain $i\left(g_{i}\right)=$ $p+1, \rho_{p}\left(g_{i}\right)=+\infty, \rho_{p+1}\left(g_{i}\right)=\rho_{p}\left(A_{0}\right)$ with at most one exceptional solution $g_{0}$ satisfying $\rho_{p+1}\left(g_{0}\right)<\rho_{p}\left(A_{0}\right)$. Let $g_{i}$ be a solution of (2.23) such that $i\left(g_{i}\right)=p+1, \rho_{p}\left(g_{i}\right)=+\infty$, $\rho_{p+1}\left(g_{i}\right)=\rho_{p}\left(A_{0}\right)$ and let $\varphi(z)(\not \equiv 0)$ be a meromorphic function satisfying $i(\varphi)<p$ or $\rho_{p+1}(\varphi)<\rho_{p}\left(A_{0}\right)$. Now, set $w(z)=g_{i}(z)+\varphi(z)$. Then

$$
i(w)=i\left(g_{i}\right)=p+1, \rho_{p+1}(w)=\rho_{p+1}\left(g_{i}\right)=\rho_{p+1}(f)=\rho_{p}\left(A_{0}\right) .
$$

On other hand, by using the fact that $g_{i}=w+\varphi$ and Lemma 2.13, we get

$$
A_{k}^{i}(z) w^{(k)}+A_{k-1}^{i}(z) w^{(k-1)}+\cdots+A_{0}^{i}(z) w=D_{i}(z),
$$

where

$$
D_{i}(z)=F^{i}(z)-\left(A_{k}^{i}(z) \varphi^{(k)}+A_{k-1}^{i}(z) \varphi^{(k-1)}+\cdots+A_{0}^{i}(z) \varphi\right) .
$$


Now, we prove that $\rho_{p}\left(D_{i}\right)=+\infty$. By $\rho_{p}\left(\frac{1}{A_{0}^{i-1}}\right)=\rho_{p}\left(A_{0}^{i-1}\right)<+\infty, \rho_{p}\left(F^{i-1}\right)=+\infty$ and Lemma 2.11, we get $\rho_{p}\left(\frac{F^{i-1}}{A_{0}^{i-1}}\right)=+\infty$. By Lemma 2.12, we have

$$
\rho_{p}\left(A_{0}^{i-1}\left(\frac{F^{i-1}}{A_{0}^{i-1}}\right)^{\prime}\right)=\rho_{p}\left(\left(\frac{F^{i-1}}{A_{0}^{i-1}}\right)^{\prime}\right)=+\infty .
$$

Since

$$
A_{0}^{i-1}(z)\left(\frac{F^{i-1}}{A_{0}^{i-1}(z)}\right)^{\prime}=\left(F^{i-1}(z)\right)^{\prime}-\frac{\left(A_{0}^{i-1}(z)\right)^{\prime}}{A_{0}^{i-1}(z)} F^{i-1},
$$

then, we get

$$
\rho_{p}\left(\left(F^{i-1}(z)\right)^{\prime}-\frac{\left(A_{0}^{i-1}(z)\right)^{\prime}}{A_{0}^{i-1}(z)} F^{i-1}\right)=+\infty .
$$

Suppose that $\rho_{p}\left(D_{i}(z)\right)<+\infty$. Then by (3.16), we have

$$
\begin{gathered}
\rho_{p}\left(F^{i}(z)\right)=\rho_{p}\left(\left(F^{i-1}(z)\right)^{\prime}-\frac{\left(A_{0}^{i-1}(z)\right)^{\prime}}{A_{0}^{i-1}(z)} F^{i-1}\right) \\
=\rho_{p}\left\{D_{i}(z)+\left(A_{k}^{i}(z) \varphi^{(k)}+A_{k-1}^{i}(z) \varphi^{(k-1)}+\cdots+A_{0}^{i}(z) \varphi\right)\right\} \\
\leq \max \left(\rho_{p}\left(D_{i}(z)\right), \rho_{p}\left\{\left(A_{k}^{i}(z) \varphi^{(k)}+A_{k-1}^{i}(z) \varphi^{(k-1)}+\cdots+A_{0}^{i}(z) \varphi\right)\right\}\right) \\
\leq \max \left\{\rho_{p}\left(D_{i}(z)\right), \rho_{p}(\varphi), \rho_{p}\left(A_{j}\right)(j=0,1, \cdots, k)\right\}<+\infty .
\end{gathered}
$$

So, (3.19) contradicts with (3.18). Hence $\rho_{p}\left(D_{i}\right)=+\infty$. Since $\rho_{p+1}\left(\frac{1}{A_{0}^{i-1}}\right)=\rho_{p+1}\left(A_{0}^{i-1}\right)$, then by Lemma 2.8, we have

$$
\begin{gathered}
\rho_{p+1}\left(D_{i}(z)\right)=\rho_{p+1}\left(F^{i}(z)-\left(A_{k}^{i}(z) \varphi^{(k)}+A_{k-1}^{i}(z) \varphi^{(k-1)}+\cdots+A_{0}^{i}(z) \varphi\right)\right) \\
\leq \max \left(\rho_{p+1}\left(F^{i}\right), \rho_{p+1}\left(A_{k}^{i}(z) \varphi^{(k)}+A_{k-1}^{i}(z) \varphi^{(k-1)}+\cdots+A_{0}^{i}(z) \varphi\right)\right) \\
\leq \max \left(\rho_{p+1}(F), \rho_{p+1}(\varphi), \rho_{p+1}\left(A_{j}\right)(j=0,1, \cdots, k)\right) \\
=\rho_{p+1}(F)<\rho_{p}\left(A_{0}\right) .
\end{gathered}
$$

By (1.6), (3.14) and (3.20), we get

$$
\max \left(\rho_{p+1}\left(D_{i}\right), \rho_{p+1}\left(A_{j}^{i}\right) \quad(j=0,1, \cdots, k)\right)<\rho_{p}\left(A_{0}\right)=\rho_{p+1}(w) .
$$

So, by Lemma 2.10 we know that all solutions of (3.15) with $\rho_{p+1}(w(z))=\rho_{p}\left(A_{0}\right)$ satisfy

$$
i_{\bar{\lambda}}(w)=i_{\lambda}(w)=i(w)=p+1, \bar{\lambda}_{p+1}(w)=\lambda_{p+1}(w)=\rho_{p+1}(f)=\rho_{p}\left(A_{0}\right) .
$$

By (3.22), we get $i_{\bar{\lambda}}\left(f^{(i)}-\varphi\right)=i_{\lambda}\left(f^{(i)}-\varphi\right)=p+1, \bar{\lambda}_{p+1}\left(f^{(i)}-\varphi\right)=\lambda_{p+1}\left(f^{(i)}-\varphi\right)=$ $\rho_{p+1}(f)=\rho_{p}\left(A_{0}\right)$, with at most one exceptional solution $f_{0}$ satisfying $\rho_{p+1}\left(f_{0}\right)<\rho_{p}\left(A_{0}\right)$. 


\section{References}

[1] B. Belaïdi, K. Hamani, Order and hyper-order of entire solutions of linear differential equations with entire coefficients, Electron. J. Differential Equations 2003, No. 17, 12 pp.

[2] B. Belaïdi, S. Hamouda, Growth of solutions of $n$-th order linear differential equation with entire coefficients, Kodai Math. J. 25 (2002), no. 3, 240-245.

[3] B. Belaïdi, Iterated order of meromorphic solutions of homogeneous and non-homogeneous linear differential equations, Romai J.,v. 11, No.1 (2015), 33-46.

[4] B. Belaïdi, On the [p, q]-order of meromorphic solutions of linear differential equations, Acta Univ. M. Belii Ser. Math. 2015, 37-49.

[5] T. B. Cao, Z. X. Chen, X. M. Zheng and J. Tu, On the iterated order of meromorphic solutions of higher order linear differential equations, Ann. Differential Equations 21 (2005), no. 2, 111-122.

[6] Y. Chen, The growth and fixed points of solutions of a type of second order nonhomogeneous differential equations, Math. Appl. (Wuhan) 29 (2016), no. 1, 117-124.

[7] Z. X. Chen, C. C. Yang, Some further results on the zeros and growths of entire solutions of second order linear differential equations, Kodai Math. J. 22 (1999), no. 2, 273-285.

[8] A. El Farissi, On the iterated exponent of convergence of solutions of linear differential equations, Int. J. Anal. Appl., Volume 7, Number 2 (2015), 162-170.

[9] A. Ferraoun and B. Belaïdi, Growth of solutions of complex differential equations with coefficients being Lacunary series of finite iterated order, Nonlinear Studies, Vol. 23, No 2 (2016), 237-252.

[10] A. A. Goldberg, I. V. Ostrovskii, The distribution of values of meromorphic functions, Irdat Nauk, Moscow, 1970 (in Russian), Transl. Math. Monogr., vol. 236, Amer. Math. Soc., Providence RI, 2008.

[11] G. G. Gundersen, Estimates for the logarithmic derivative of a meromorphic function, plus similar estimates, J. London Math. Soc. (2) 37 (1988), no. 1, 88-104.

[12] G. G. Gundersen, Finite order solutions of second order linear differential equations, Trans. Amer. Math. Soc. 305 (1988), no. 1, 415-429.

[13] W. K. Hayman, Meromorphic functions, Oxford Mathematical Monographs Clarendon Press, Oxford 1964.

[14] J. He, X. M. Zheng and H. Hu, Iterated order of meromorphic solutions of certain higher order linear differential equations with meromorphic coefficients of finite iterated order, Acta Univ. Apulensis Math. Inform. No. 33 (2013), 145-157.

[15] L. Kinnunen, Linear differential equations with solutions of finite iterated order, Southeast Asian Bull. Math. 22 (1998), no. 4, 385-405.

[16] I. Laine, Nevanlinna theory and complex differential equations, de Gruyter Studies in Mathematics, 15. Walter de Gruyter \& Co., Berlin, 1993.

[17]J. R. Long, J. Zhu, On hyper-order of solutions of higher order linear differential equations with meromorphic coefficients, Adv. Difference Equ. 2016, 2016:107, 13 pp. 
[18] M. Saidani, B. Belaïdi, On the fast growth of solutions to higher order linear differential equations with entire coefficients, Novi Sad J. Math. 46 (2016), no. 2, 117-133.

[19] J. Tu, Z. X. Chen, Growth of solutions of complex differential equations with meromorphic coefficients of finite iterated order, Southeast Asian Bull. Math. 33 (2009), no. 1, 153-164.

[20] J. Tu, T. Long, Oscillation of complex high order linear differential equations with coefficients of finite iterated order, Electron. J. Qual. Theory Differ. Equ. 2009, No. 66, $1-13$.

[21] L. Yang, Value Distribution Theory, Springer-Verlag, Berlin, 1993.

[22] C. C. Yang, H. X. Yi, Uniqueness theory of meromorphic functions, Mathematics and its Applications, 557. Kluwer Academic Publishers Group, Dordrecht, 2003. 